\title{
The Role of Insertion Points in the Detection and Positioning of Instruments in Laparoscopy for Robotic Tasks
}

\author{
Christophe Doignon, Florent Nageotte, and Michel de Mathelin \\ Control, Vision and Robotic Team, \\ LSIIT (UMR ULP-CNRS 7005) University of Strasbourg, France \\ name@lsiit.u-strasbg.fr
}

\begin{abstract}
In robot-assisted laparoscopic surgery, an endoscopic camera is used to control the motion of surgical instruments. With this minimally invasive surgical (MIS) technique, every instrument has to pass through an insertion point in the abdominal wall and is mounted on the endeffector of a surgical robot which can be controlled by visual feedback. To achieve an accurate vision-based positioning of laparoscopic instruments, we introduce the motion constraint in MIS which is based on the location of out-of-field of view insertion points. The knowledge of the (image of the) insertion point location is helpful for real-time image segmentation issues, particularly to initiate the search for region seeds corresponding to the instruments. Moreover, with this "eye-to-hand" robot vision system, visual servoing is a very convenient technique to automatically guide an instrument but it requires the velocity screw to be expressed in the appropriate frame. Then, the location of the insertion point is seen as the main part of the larger problem of determining the overall transformation between the camera and the robot end-effector frame. This is achieved thanks to a novel algorithm for the pose determination of cylindricalshaped instruments. With the proposed method, the location of insertion points can be recovered, on-line, with no marker, without any knowledge of robot kinematics and without an external measurement device.
\end{abstract}

\section{Introduction and Motivations}

In laparoscopic surgery, motions of surgical instruments are constrained to by the insertion point locations in the abdominal wall [1, reducing the mobility since only 4 degrees of freedom are available (see Fig. 1] (left)). Endoscopic vision systems may be used for intra-operative guidance of instruments, however to achieve an accurate positioning inside the abdominal cavity, many hard problems must be overcome. Well-known difficulties are emanating from the timevarying lighting conditions, the presence of specularities, bloodstained parts, smoke and a non-uniform and moving background due to patient breathing and heart beating. The major problem for this "eye-to-hands" robot vision system is the unknown position/orientation of the instrument w.r.t. the camera frame which must be recovered to properly guide instruments thanks to visual servoing techniques. Visual servoing consists of using the data provided by cameras to 

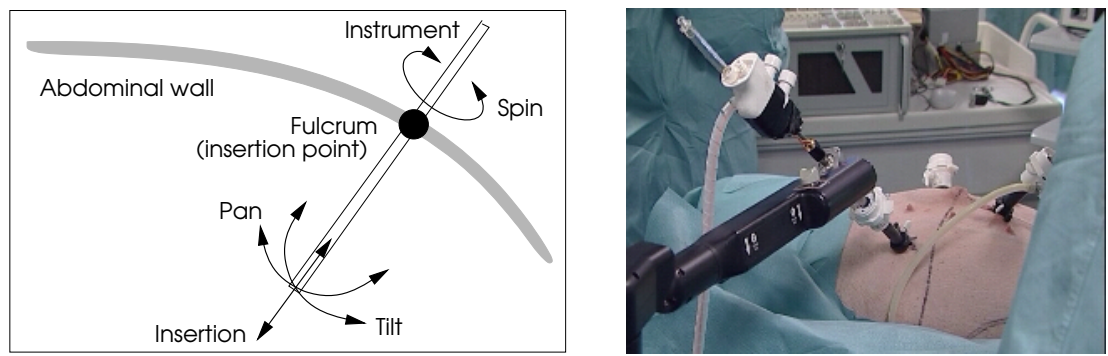

Fig. 1. (left) The free 4 DOFs (insertion/pan/tilt/spin) in laparoscopic surgery. (right) The instrument mounted onto the AESOP surgical robot in the operating room.

control the motion of a robot [2. To this aim, a set of visual features $\mathbf{s}$ must be designed from the data measurements to control the desired degrees of freedom (DOF). To perform an image-based visual servoing, one needs to compute an interaction matrix $\mathrm{L}_{s}$, which links the time variations of visual features to the relative camera-object kinematics screw $\boldsymbol{\tau}$ which is defined by $\dot{\mathbf{s}}=\mathrm{L}_{\mathbf{s}} \boldsymbol{\tau}[3$. To control the robot using the joint velocities, we must have $\dot{\mathbf{s}}=\mathrm{J}_{s} \dot{\mathbf{q}}+\frac{\partial \mathbf{s}}{\partial t}$, and $\mathrm{J}_{\mathrm{s}}=-\mathrm{L}_{\mathrm{s}}{ }^{c} \mathrm{~T}_{i}{ }^{i} \mathrm{~T}_{e}{ }^{e} \mathrm{~J}(\mathbf{q})$, where ${ }^{e} \mathrm{~J}(\mathbf{q})$ is the robot Jacobian expressed in the endeffector frame $\left(R_{e}\right) .{ }^{c} \mathrm{~T}_{i}$ is the time-varying transformation between the camera frame $\left(R_{c}\right)$ and the instrument frame $\left(R_{I}\right)$. With the 4 DOFs available in MIS, if $\mathbf{v}$ is the vector corresponding to the penetration direction, it is expressed with

$$
{ }^{c} \mathrm{~T}_{i}=\left[\begin{array}{cc}
\mathbf{v} & {[\mathbf{t}]_{\times} \mathrm{R}} \\
\mathbf{0}_{3} & \mathrm{R}
\end{array}\right],
$$

where $\mathrm{R}=\left(\mathbf{r}_{1}, \mathbf{r}_{2}, \mathbf{v}\right)$ is the rotation matrix and $\mathbf{t}$ is the translation vector between the camera frame $\left(R_{c}\right)$ and the instrument frame $\left(R_{I}\right)$. The estimation of vectors $\mathbf{v}$ and $\mathbf{t}$ is the main objective and contribution of the paper. The organization is as follows. In the next section, we review some endoscopic vision systems used in robot-assisted laparoscopy. In section three, we briefly present the image segmentation, we formalize the motion constraint together with the $3 \mathrm{D}$ pose of cylindrical-shaped objects. In section four, we present experimental results for the location of the insertion point in surgical conditions.

\section{Related Work on Vision-Based Robotic Guidance for Minimally Invasive Abdominal Surgery}

Prior works have been conducted to process laparoscopic images for the development of navigation systems in the human body. Wei et al. 4 have used a laparoscope mounted on a robot arm and have designed a color marker to realize a tracking task. This spectral mark was then utilized to control the robot motion at a sampling rate of $15 \mathrm{~Hz}$. Wang et al. [5] have proposed to enhance laparoscope manoeuvering capabilities. In so doing, they have conceived a general framework to assist the surgeon in manipulating a laparoscope mounted on 
a robot end-effector. This framework has been applied to the $2 \mathrm{D}$ positioning of the imaged tip of instrument with the AESOP robot in a way to follow the laparoscope. Like for the previous related work, it's a $2 \mathrm{D}$ visual tracking system.It is also assumed that the camera is mounted on a robot (eye-in-hand). Other more recent works are rather related to the 3D tracking with a stationary camera. Hayashibe et al. 6] have designed an active scanning system with structured lighting for the reconstruction of 3D intraoperative local geometry of pointed organs. A robot vision system that automatically positions a single laparoscopic instrument with a stationary camera is described by Krupa et al. 7]. A laser pointing device has been designed to emit markers on the organ. A visual servoing algorithm is carried out to position a marked instrument by combining pixel coordinates of the laser spots and the estimated distance between the pointed organ surface and the tip of the instrument. It is worth noticing that a on-line identification of the Jacobian matrix for pan/tilt control was realized with robot joint motions to directly get expressions of the velocity screw in the instrument frame. At the Center for Computer Integrated Surgical Systems and Technology (CISST), several techniques for assisting surgeons have been developed. Many of them involve (mono- and stereo-) vision-based robot control with articulated instruments 8]. To identify the robot (fixed) frame-to-camera transformation, the Optrotrak system is used. Burschka et al. have noticed an offset of approximately $5 \mathrm{~mm}$ which is due to the difficulty of segmenting led centers.

Some solutions for the recovery of insertion points have been provided by Krupa et al. [7] and also by Ortmaier et al. [9] but with respect to the robot frame, which inherently introduces errors of the robot model. Moreover, these methods need specific markers. Robotic tasks may require interactions with tissues, instruments must be autoclavable before a surgical operation and since several one may alternatively be used (depending on the subtask addressed), it is not convenient to always use artificial landmarks placed on endoscopic tools.

\section{Localization of Instruments and Motion Constraint}

In this section, we formalize the motion constraint. A scene structure from motion approach is developed to get the location of insertion points. To this aim, the automatic segmentation of instruments is briefly presented and a novel algorithm for the 3D pose estimation of cylindrical-shaped instrument is detailled.

\subsection{The Motion Constraint in MIS}

The multiview approach we present exploits existing motion constraints of instruments observed by a stationary camera. We denote with $\left(R_{c}\right)$ the camera frame with projection centre $\mathbf{C},\left(R_{I}\right)$ the reference frame attached to a laparoscopic instrument with an arbitrary origin $\mathbf{O}_{I}$. We define a unit vector $\mathbf{v}$ as the direction of instrument axis. The small incision area in the abdominal wall is represented with a geometrical point $\mathbf{I}$ (Fig. 2 (left)). With the notations used in (1), we have $\mathbf{C I}=\mathbf{t}+\mathrm{R} \mathbf{O}^{I} \mathbf{I}=\mathbf{t}+\lambda \mathrm{R}\left[\begin{array}{lll}0 & 0 & 1\end{array}\right]^{\top}=\mathbf{t}+\lambda \mathbf{v}$, for any 

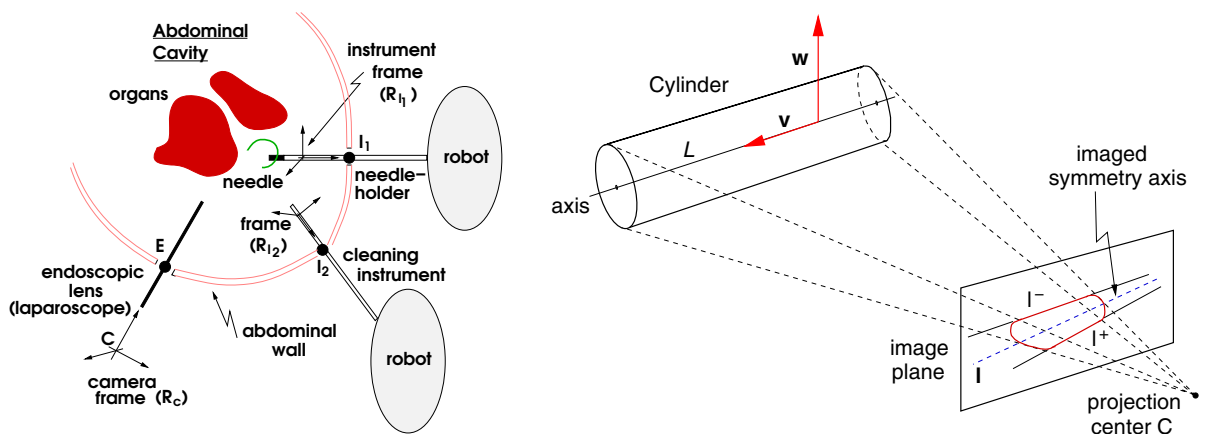

Fig. 2. (left) In laparoscopy, endoscopic camera can observe organs and parts of instruments with motions constrained to insertion points $I_{1}, I_{2}, \ldots$ on the abdominal wall. (right) Plücker coordinates of the cylinder axis and its perspective projection.

scalar $\lambda \in \mathbb{R}$. Since most instruments exhibit a surface of revolution (SOR), the attitude of the axis of revolution may conveniently be represented with the Plücker coordinates as it is for any $3 \mathrm{D}$ straight line $L$. It is a couple of algebraically dependent vectors $(\mathbf{v}, \mathbf{w})$ such that $\mathbf{v}^{\top} \mathbf{w}=0$. Thus $\mathbf{w}$ is chosen as the vector normal to the interpretation plane of the viewed line, that is the plane passing through the projection center and the 3D line (see Fig. 2 (right)). They may alternatively be gathered in the following (singular) matrix $\mathrm{L}$ or its dual $\mathrm{L}^{\star}$ $\left(\mathrm{L} \mathrm{L}^{\star}=0\right)$ :

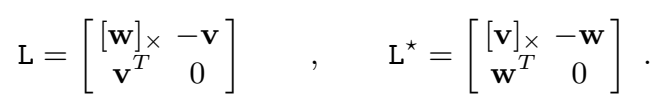

The motion constraint can be expressed as the intersection of multiple convergent 3D straight lines. Since any (homogeneous) point $\mathbf{X}$ is on $L$ if $\mathrm{L}^{\star} \mathbf{X}=\mathbf{0}$, given $n$ displacements corresponding to the set of dual Plücker matrices $\left\{\mathrm{L}_{1}^{\star}, \mathrm{L}_{2}^{\star}, \ldots, \mathrm{L}_{n}^{\star}\right\}$, the intersection of lines is obtained with a rank-3 $(4 n \times 4)$ matrix $\mathrm{G}_{n}^{\top}$ such that $\mathrm{G}_{n}=\left[\mathrm{L}_{1}^{\star}, \mathrm{L}_{2}^{\star}, \ldots, \mathrm{L}_{n}^{\star}\right]$. That is, the null-space of $\mathrm{G}_{n}^{\top}$ must be a one-dimensional subspace and the intersection may be computed with $n(n \geq 2) 3 \mathrm{D}$ displacements. By computing the SVD of $G_{n}^{\top}$, one obtains the common intersection with the singular vector associated to the null singular value. Moreover, the perspective projection of the $3 \mathrm{D}$ line $L_{j}$ is the image line $\mathbf{l}_{j}$ defined by (see [10, p. 186)

$$
\left[\mathbf{l}_{j}\right]_{\times}=\mathrm{K}^{c} \mathrm{P}^{c} \mathrm{~L}_{j}\left(\mathrm{~K}^{c} \mathrm{P}^{c}\right)^{\top}=\left[\left(\mathrm{K}^{c}\right)^{-\mathrm{T}} \mathbf{w}_{j}\right]_{\times} \Rightarrow \mathbf{l}_{j} \equiv\left(\mathrm{K}^{c}\right)^{-\mathrm{T}} \mathbf{w}_{j},
$$

where $\mathrm{K}^{c}$ is the matrix of camera parameters and $\mathrm{P}^{c}$ is the projection matrix. Since vector $\mathbf{l}_{j}$ is defined up to a scale, it does not depend on the magnitude of vector $\mathbf{w}_{j}$, hence the $n$ convergent image lines $\mathbf{l}_{1}, \mathbf{l}_{2}, \ldots, \mathbf{l}_{n}$ must satisfy

$$
\left(\mathbf{l}_{1} \mathbf{l}_{2} \ldots \mathbf{l}_{n}\right)^{\top} i=\underbrace{\left(\mathbf{w}_{1} \mathbf{w}_{2} \ldots \mathbf{w}_{n}\right)^{\top}}_{\mathbf{w}_{n}}\left(\mathrm{~K}^{c}\right)^{-1} i=\mathbf{0}
$$

where $i$ is the image of the insertion point $I$. It follows that a set of $n 3 \mathrm{D}$ straight lines is projected to $n$ convergent image lines if the above $(n \times 3)$ matrix $\mathrm{W}_{n}$ is 
of rank 2. It's only a necessary condition which does not ensure the convergence of the 3D lines, but which makes so important the accurate estimation of the imaged cylinder axis (lines $\mathbf{l}_{j}$ ), hence the estimation of its Plücker coordinates.

\subsection{Real-Time Segmentation in the Abdominal Cavity}

In laparoscopic surgery, many surgical instruments have metallic parts leading to grey regions in the image. The color image segmentation we designed is based on chromatic HS (Hue-Saturation) color attributes. The joint color feature $\mathcal{H}=S H$ is a powerful discriminant cue as shown in Fig. 3 (middle). To get out an oversegmentation, a fast non linear filtering has been especially designed. A region-based segmentation approach has been developed, and since any instrument is constrained to pass through the insertion point, the automatic detection of seeds to initiate a region growing process is reduced to a one-dimensional search of low $\mathcal{H}$ values along the image boundaries. The region boundary-pixels are then extracted and classified in two classes and a robust least median of squares method is used for modelling the apparent contour of cylindrical instrument with a pair of lines $\left(\mathbf{l}^{-}\right.$and $\mathbf{l}^{+}$, Fig. 3 (bottom)), or to reject the region if the distance between boundary-pixels and the corresponding (putative) line is too large (see [1] for details).

\subsection{Pose Computation of a Cylinder}

We present a novel algorithm for the pose estimation of a cylinder. As a close related work, Wong et al. 12 have proposed an algorithm which is able to recover the orientation and the depth while an image rectification is performed to coincide the imaged revolution axis of a SOR with one image axis and when the image of a latitude circle is available. The method we propose here is designed
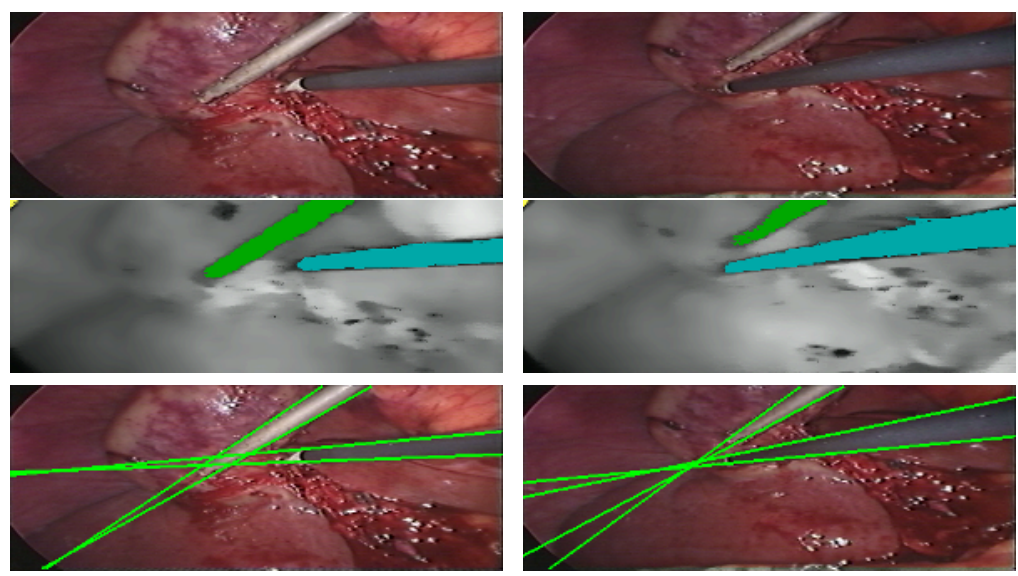

Fig. 3. Segmentation of surgical instruments in the abdominal cavity for two endoscopic images. (top) Original images. (middle) Selected regions in the HS-image. (bottom) Estimation of the pair of straight lines from the apparent contours (in green). 
for straight circular cylinders. All components are computed in one stage and it does not need any image transformation and no latitude circle, hence it can deal with partial occlusion of the apparent contour as it is for this application area. Given the radius $r_{c}$ and the apparent contour, we look for the Plücker coordinates $(\mathbf{v}, \mathbf{w})$ of the cylinder axis. It can be shown with the perspective model that any point $m$ lying on the apparent lines $\mathbf{l}^{-}$and $\mathbf{l}^{+}$must satisfy

$$
\begin{aligned}
\left(\mathbf{I}^{-}\right)^{\top} m & \equiv\left\{\left(\mathrm{K}^{c}\right)^{-\mathrm{\top}}\left(\mathrm{I}-\alpha[\mathbf{v}]_{\times}\right) \mathbf{w}\right\}^{\top} m=0 \\
\left(\mathbf{I}^{+}\right)^{\top} m & \equiv\left\{\left(\mathrm{K}^{c}\right)^{-\mathrm{\top}}\left(\mathrm{I}+\alpha[\mathbf{v}]_{\times}\right) \mathbf{w}\right\}^{\top} m=0,
\end{aligned},
$$

with $\alpha=r_{c} / \sqrt{\|\mathbf{w}\|^{2}-r_{c}^{2}}$. To compute the pose parameters, we define vectors $\mathbf{y}=\alpha[\mathbf{v}]_{\times} \mathbf{w}, \boldsymbol{\rho}^{-}=\left(\mathrm{K}^{c}\right)^{\top} \mathbf{l}^{-}$and $\boldsymbol{\rho}^{+}=\left(\mathrm{K}^{c}\right)^{\top} \mathbf{l}^{+}$. With these notations, (5) becomes

$$
\left[\begin{array}{rccc}
-\mathrm{I} & \mathrm{I} & -\boldsymbol{\rho}^{-} & \mathbf{0} \\
\mathrm{I} & \mathrm{I} & \mathbf{0} & -\boldsymbol{\rho}^{+}
\end{array}\right]\left[\mathbf{y}^{\top} \mathbf{w}^{\top} \mu_{1} \mu_{2}\right]^{\top}=\mathrm{A}_{6 \times 8} \quad \mathbf{x}=\mathbf{0}
$$

with the unkwown vector $\mathbf{x}=\left(\mathbf{y}^{\top}, \mathbf{w}^{\top}, \mu_{1}, \mu_{2}\right)^{\top}$. and $\mu_{1}$ and $\mu_{2}$ are two non-null scalars. $\mathbf{y}$ and $\mathbf{w}$ are algebraically dependent since they satisfy $\mathbf{y}^{\top} \mathbf{w}=0$ and $\|\mathbf{y}\|=|\alpha|\|\mathbf{w}\|$. The latter equation should be developed so as to extract the expression for $\alpha$

$$
r_{c}^{2}\left(\|\mathbf{w}\|^{2}+\|\mathbf{y}\|^{2}\right)=\|\mathbf{w}\|^{2}\|\mathbf{y}\|^{2}
$$

Since $\mathrm{A}_{6 \times 8}$ has a rank equal to 6 , the $\operatorname{SVD} \mathrm{U}_{6 \times 8} \mathrm{D}\left(\mathbf{v}_{1}, \cdots, \mathbf{v}_{8}\right)^{\top}$ has two null singular values and the null-space of $\mathrm{A}_{6 \times 8}$ is spanned by the right singular vectors $\mathbf{v}_{7}$ and $\mathbf{v}_{8}$ and provides a 2-parameter family of solutions as a linear combination of the two last columns of $\mathrm{V}$ as $\mathbf{x}=\lambda \mathbf{v}_{7}+\tau \mathbf{v}_{8}, \quad$ for $\lambda, \tau \in \mathbb{R}$. Then, substituting $\mathbf{y}=\left(x_{1}, x_{2}, x_{3}\right)^{\boldsymbol{\top}}$ and $\mathbf{w}=\left(x_{4}, x_{5}, x_{6}\right)^{\boldsymbol{\top}}$ in $\mathbf{y}^{\boldsymbol{\top}} \mathbf{w}=0$ gives the following homogeneous quadratic equation in $\lambda$ and $\tau a_{1} \lambda^{2}+a_{2} \lambda \tau+a_{3} \tau^{2}=0$, where $a_{i}$ are scalar functions of $\mathbf{v}_{7}$ and $\mathbf{v}_{8}$. Two real solutions for $s=\tau / \lambda$, $s^{-}$and $s^{+}$, can be computed. Then, reporting these solutions in (7) gives an homogeneous quadratic equation $c_{1}(s) \tau^{2}+c_{2}(s) \tau^{4}=0$ and the solutions are $\tau=0$ (double) and $\tau= \pm \sqrt{-\frac{c_{1}(s)}{c_{2}(s)}}$.

\section{Experimental Results}

With a training box at the lab, the detection and tracking of a surgical instrument has carried out (see Fig. 团(top)) with the AESOP surgical robot. During the guidance, we noticed some small temporal variations of the image $\left(i_{1}\right)$ of the insertion point $I_{1}$ due to errors in the overall segmentation (Fig. 4 (bottom middle)). In Fig. 团(bottom right), we have reported the dual parameter space of convergent lines $\mathbf{l}_{j}$ (distance versus angle of image line direction), since a unique intersection of lines must lead to perfectly collinear points (blue bullets).

In the operating room of IRCAD 1 (see Fig. 1)(right)), this method has been validated with a living pig. The precision of the image of the insertion point

\footnotetext{
${ }^{1}$ Institut de Recherche contre les Cancers de l'Appareil Digestif, Strasbourg, France.
} 

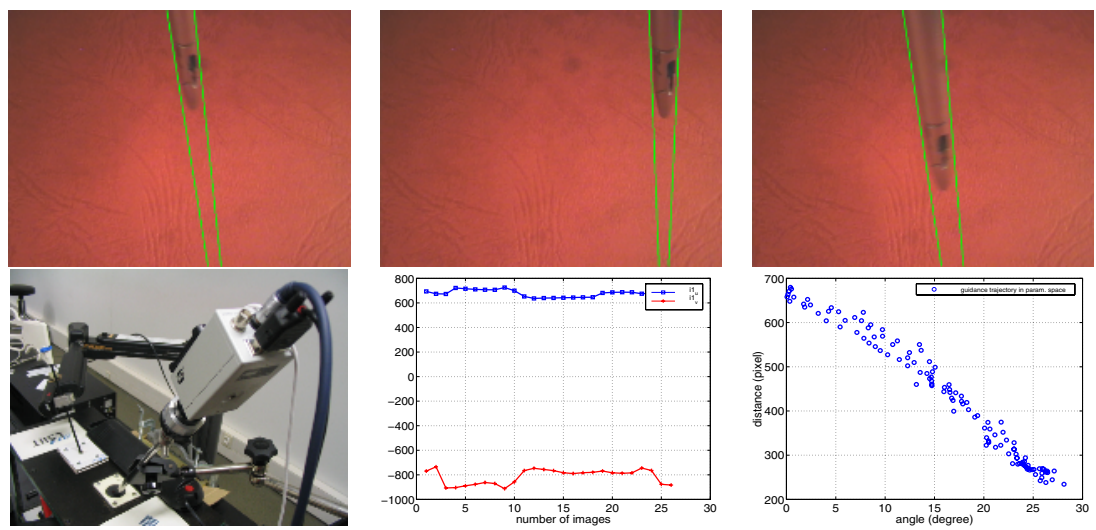

Fig. 4. Experiments in the lab to validate the proposed method. (top) Three images with the detection of a single surgical instrument (in green). (left) A training box is used together with the endoscope fixed onto a monoCCD camera. (middle) Temporal variations of $i_{1}$ coordinates in the image while moving the surgical instrument. (right) In the parameter space of convergent lines $(\theta, \rho)$ corresponding to the imaged instrument axis, "points" (blue bullets) must be collinear with a motionless insertion point.
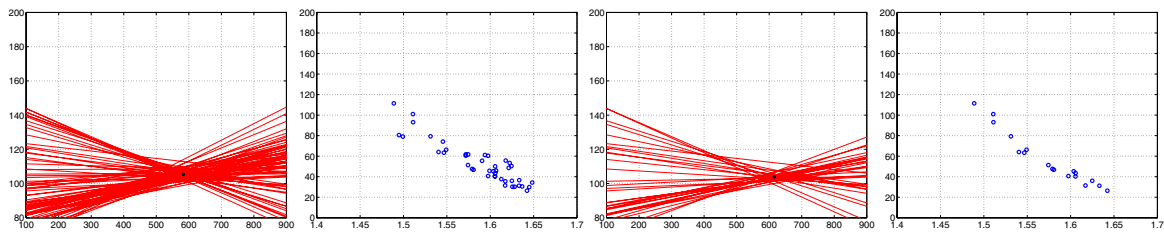

Fig. 5. (left) The convergent imaged symmetric axes and the estimated image of the insertion point $i_{1}$ at $(593.4 ; 105.5)$ (black cross) computed with the least mean squares during the guidance of an instrument in the operating room. (middle left) In the parameter space of onvergent lines $(\theta, \rho)$, "points" (blue bullets) must be collinear. (middle right) The estimated image of the insertion point $i_{1}$ at $(615.5 ; 103.9)$ (black cross) and the parameter space (right) with the robust estimation when $50 \%$ of data are rejected.

$i_{1}=(593.4 ; 105.5)$ (see Fig. 5 (left)) is given by the standard deviations $\sigma_{u}=25.4$ and $\sigma_{v}=4.1$ pixels respectively in the horizontal $(u)$ and vertical directions $(v)$ with the least mean squares method. These results show a significantly better precision in the vertical direction. This can be explained either by the breathing motion or by a no sufficient spread of orientation motions in one direction while the robot is guiding the instrument. By performing a least median of squares method, $50 \%$ of displacements have been discarded (see Fig. 5 (right)) so as to get a robust estimate of $i_{1}$. Standard deviations are then $\sigma_{u}=11.4$ pixels and $\sigma_{v}=2.6$ pixels with the robust method. Finally, the Plücker coordinates of the remaining lines are used to get the 3D location of $I_{1}=(-258.9 \pm 29.4 ;-68.4 \pm$ $5.2 ; 316.4 \pm 34.7)$ with the selected displacements. 


\section{Conclusion}

In this paper, we have proposed a method to estimate the location of out-of-field of view insertion points on the abdominal wall suited for vision-based robotic guidance in MIS. This is helpful to drive the image segmentation based on seeded region growing and it is needed for expressing the velocity screw in the appropriate frame so as to track surgical instruments with visual servoing techniques.

For cylindrical-shaped laparoscopic instruments, we have presented a novel algorithm which solves the Plücker coordinates of a cylinder axis and which can deal with partial occlusions of instruments. Despite the disturbance due to the patient breathing, we have shown that this method provides satisfactory results in real surgical conditions with only few endoscopic images.

\section{References}

1. Funda, J., Taylor, R., Eldridge, B., Gomory, S., Gruben, K.: Constrained cartesian motion control for teleoperated surgical robots. IEEE Trans. on Robotics and Automation 12 (1996)

2. Hutchinson, S., Hager, G.D., Corke, P.: A tutorial on visual servo control. IEEE Trans. on Robotics and Automation 12 (1996) 651-670

3. Espiau, B., Chaumette, F., Rives, P.: A new approach to visual servoing in robotics. IEEE Transactions on Robotics and Automation 8 (1992) 313-326

4. Wei, G.Q., Arbter, K., Hirzinger, G.: Real-time visual servoing for laparoscopic surgery. IEEE Engineering in Medicine and Biology 16 (1997) 40-45

5. Wang, Y.F., Uecker, D.R., Wang, Y.: A new framework for vision-enabled and robotically assisted minimally invasive surgery. Journal of Computerized Medical Imaging and Graphics 22 (1998) 429-437

6. Hayashibe, M., Nakamura, Y.: Laser-pointing endoscope system for intra-operative geometric registration. In: Proc. of ICRA'01, Seoul, South Korea (2001)

7. Krupa, A., Gangloff, J., Doignon, C., de Mathelin, M., Morel, G., Leroy, J., Soler, L., Marescaux, J.: Autonomous 3-d positioning of surgical instruments in robotized laparoscopic surgery using visual servoing. IEEE Trans. on Robotics and Automation 10 (2003) 842-853

8. Burschka, D., Corso, J.J., Dewan, M., Hager, G.D., Lau, W., Li, M., Lin, H., Marayong, P., Ramey, N.: Navigating inner space: 3-d assistance for minimally invasive surgery. In: Workshop Advances in Robot Vision, IEEE/RSJ Int'l Conf. on Intelligent Robots and Systems, Sendai, Japan (2004) 67-78

9. Ortmaier, T., Hirzinger, G.: Cartesian control issues for minimally invasive robot surgery. In: Proceedings of IROS'00, Takamatsu, Japan (2000)

10. Hartley, R., Zisserman, A.: Multiple view geometry in computer vision. Cambridge Univ. Press (2000)

11. Doignon, C., Graebling, P., de Mathelin, M.: Real-time segmentation of surgical instruments inside the abdominal cavity using a joint hue saturation color feature. Real-Time Imaging 11 (2005) 429-442

12. Wong, K.Y., Mendonça, P.R.S., Cipolla, R.: Reconstruction of surfaces of revolution from single uncalibrated views. Image and Vis. Computing 22 (2004) 829-836 